\title{
Temperature Dependence of Hydrogen Adsorption Isotherms
}

\author{
Stefan Tibus, Jürgen Klier, and Paul Leiderer \\ Department of Physics, University of Konstanz, 78457 Konstanz, Germany
}

In the past it has already been shown that adsorption isotherms of liquid or solid films are not described completely by the Frenkel-Halsey-Hill theory. Substrate roughness as well as thermal fluctuations have to be taken into account in understanding the adsorption behavior. The inclusion of thermal fluctuations into the adsorption theory has already been addressed and proven to provide an explanation for the deviations found in many experiments. However, a resulting temperature dependence of such isotherms has not yet been verified. In our investigations we have addressed this issue with a series of adsorption isotherms of hydrogen on gold in a temperature range from $11 \mathrm{~K}$ to $19.5 \mathrm{~K}$ (i.e., below and above the triple-point temperature of hydrogen). Our measurements are compared with existing theories and the nature of the remaining discrepancies is discussed.

PACS numbers: $68.08 \mathrm{Bc}, 68.15+e, 68.43-h$

\section{INTRODUCTION}

The adsorption of thin films on solid surfaces is an important phenomenon which has been studied for a long time. Its qualitative features are described in terms of the well-established model by Frenkel, Halsey and Hill $(\mathrm{FHH}),{ }^{1-3}$ which relies on the van der Waals interaction between adsorbate and substrate. A careful comparison with recent experiments reveals, however, that the details of the observations are not accounted for by the FHH model. This discrepancy is the topic of the present paper.

The approach by FHH may be understood by considering an ideal gas in the presence of a wall. Under the assumption of pure van der Waals forces, as described by the Lennard-Jones potential, a gas molecule at distance $d$ 
feels an effective attractive potential

$$
U_{\mathrm{vdW}}=-\frac{\Delta C_{3}}{d^{3}}
$$

where $\Delta C_{3}$ is the Hamaker constant.

In the absence of a wall, the difference in chemical potential, $\Delta \mu$, for a molecule in the gas phase, $\mu_{\text {gas }}$, compared to a molecule in the condensed phase, $\mu_{\text {liq }}$, is given by

$$
\Delta \mu=\mu_{\text {gas }}-\mu_{\text {liq }}=-k_{\mathrm{B}} T \ln \left[\frac{p}{p_{\text {sat }}}\right]
$$

where $T$ is the temperature, and $p$ and $p_{\text {sat }}$ - the non-saturated and saturated vapor pressure, respectively. In the vicinity of a wall, Eq. (1) has to be added, leading to

$$
\Delta \mu_{\mathrm{wall}}=\Delta \mu+\mu_{\mathrm{vdW}}=-k_{\mathrm{B}} T \ln \left[\frac{p}{p_{\mathrm{sat}}}\right]-\frac{\Delta C_{3}}{d^{3}} .
$$

The FHH adsorption isotherm is now determined by equating the chemical potential at the film surface with that of a bulk liquid, i.e. $\Delta \mu_{\text {wall }}=0$, so the resulting film thickness is

$$
d=\left\{\frac{\Delta C_{3}}{-k_{\mathrm{B}} T \ln \left[p / p_{\mathrm{sat}}\right]}\right\}^{1 / 3}=\left[\frac{\Delta C_{3}}{\Delta \mu}\right]^{1 / 3} .
$$

When approaching saturated vapor pressure $\left(p \rightarrow p_{\text {sat }}\right)$ the film thickness diverges, which corresponds to the case of bulk liquid.

Divergency can, however, occur only when film and bulk are on the same level within the gravitation potential. For the case of the film being at a distance $h$ above the bulk liquid, the term

$$
\mu_{\text {grav }}=m g h
$$

has to be added to (3), where $m$ is the mass of a single molecule, and $g$ - the acceleration due to gravity. This limits the film thickness to a finite value.

As can be seen from Eq. (4), the film thickness is a function of $\Delta \mu$ only and, by choosing $p / p_{\text {sat }}$ as independent variable, it is proportional to $T^{-1 / 3}$. Fig. 1 shows some calculated curves for different temperatures. With higher temperature the film thickness decreases for the same reduced pressure $p / p_{\text {sat }}$, but it would increase for stronger attraction by the substrate (see Eq. (4)). 

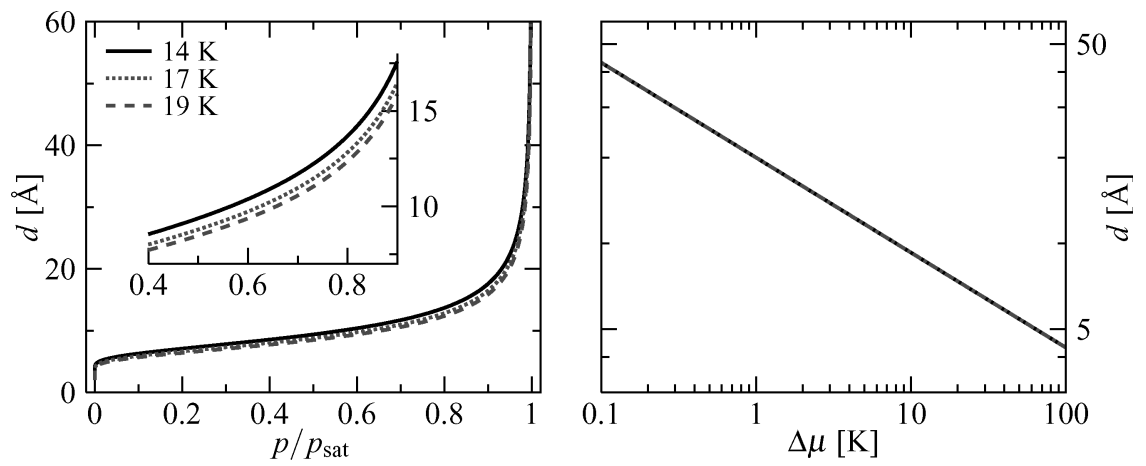

Fig. 1. Calculated adsorption isotherms according to the FHH theory, shown for different temperatures in $\mathrm{K}$ and a typical $\Delta C_{3}=8000 \mathrm{~K} \AA^{3}$. Left: The dependence $d$ versus $p / p_{\text {sat }}$ is shown. The film thickness decreases with higher temperature for the same $p / p_{\text {sat }}$. For clarity the inset shows details of the different adsorption isotherms. Right: The same in $\log d$ versus $\log \Delta \mu$, there is no temperature dependence.

\section{THEORY}

\subsection{Thermal Fluctuations}

Reference 4 extended the basic approach of molecules in a van der Waals potential by introducing thermal fluctuations. The method basically is the solid-on-solid model, considering a lattice of adsorption sites $i$ and a number $n_{i}$ of stacked molecules on each of these. The effective film thickness is the average of all local thicknesses $d_{i}=a n_{i}$, where $a$ is the size of a single adsorbate molecule. Since the film surface is now undulated the surface energy contributes to the Hamiltonian. The mean film thickness is then calculated via statistics and mean-field approximation for neighbor interactions, so it cannot be represented analytically but has to be calculated numerically.

Figure 2 shows results of calculation for two surface tensions $\gamma$ at different temperatures. It is worth to note that for small values of $p / p_{\text {sat }}$ the film is always thinner than according to FHH. With increasing temperature the film thickness at the upper end of the isotherm $\left(p / p_{\text {sat }} \rightarrow 1\right)$ increases beyond the FHH value. Higher values of surface tension suppress the fluctuations and thus the increase in thickness.

There seems to be a problem with the above theory ${ }^{4}$ as applied to hydrogen data in Refs. 5,6. The fits appear to be good only if done at higher temperatures and calculated back to reduced pressure with the actual temperature in the experiment, see Ref. 7. Furthermore, the obtained value for the surface tension is $0.058 \mathrm{~K} \AA^{-2}$, i.e. about 40 times smaller than the literature value of $2.17 \mathrm{~K} \AA^{-2}{ }^{6}$ 

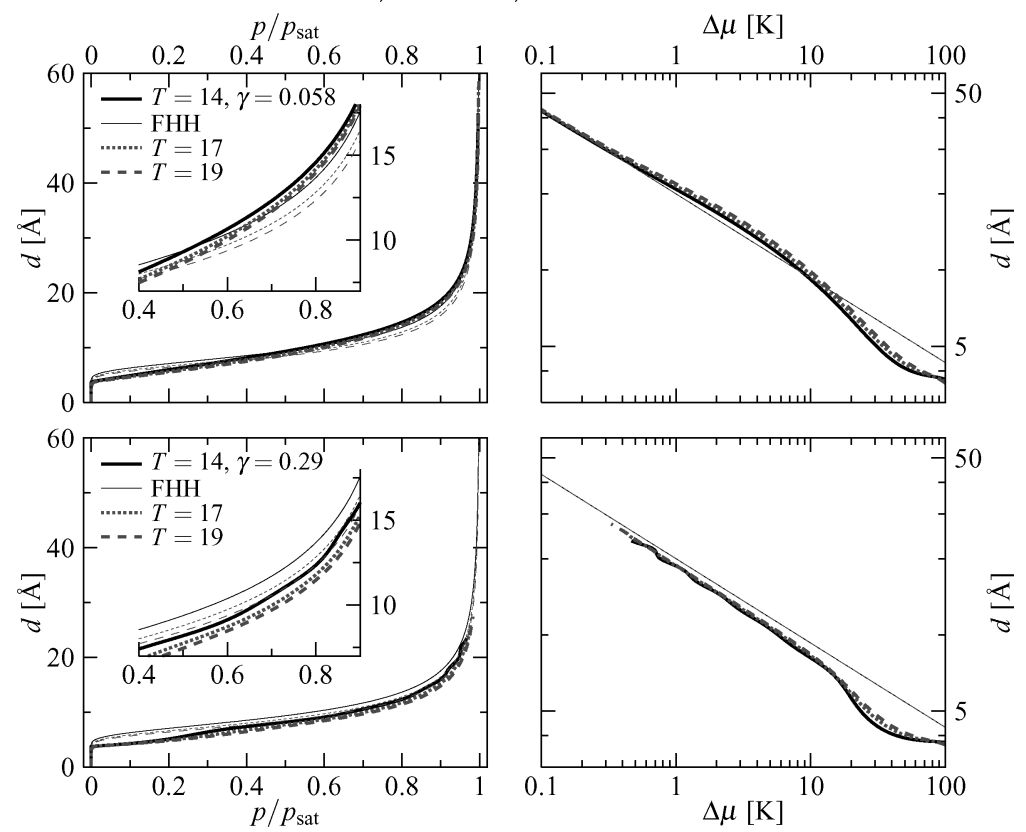

Fig. 2. Calculated adsorption isotherms according to Ref. 4 (thick lines) and FHH (thin lines) for comparison. All graphs are for $\Delta C_{3}=8000 \mathrm{~K} \AA^{3}, T$ in $\mathrm{K}$, and $\gamma$ in $\mathrm{K} \AA^{-2}$. For $p / p_{\text {sat }}<0.4$ the film is thinner than that according to $\mathrm{FHH}$, for larger values the behavior depends on $\gamma$. For small $\gamma$ (top) the film thickness increases beyond FHH at $p / p_{\text {sat }}=0.4$ to 0.5 , for higher $\gamma$ (bottom) the thickness remains below the FHH value.

\subsection{Substrate Roughness}

Another possible contribution to deviations from FHH theory arises from substrate roughness. For helium films the effect of substrate roughness has already been modelled ${ }^{8}$ and may be applied to our hydrogen films as well. Under the assumptions of negligible retardation due to the small film thickness, negligible contribution of gravitation, small curvatures $\nabla d$, and with $\Delta \mu=-k_{\mathrm{B}} T \ln p / p_{\text {sat }}$ we have

$$
\gamma V_{\mathrm{a}} \nabla^{2} d+\frac{\Delta C_{3} k_{\mathrm{B}}}{(d-\delta)^{3}}=\Delta \mu=-k_{\mathrm{B}} T \ln \left[\frac{p}{p_{\mathrm{sat}}}\right]
$$

where $V_{\mathrm{a}}$ is the volume of a single adsorbate molecule, $\delta$ - the amplitude of the roughness peaks of the substrate, and $d=d_{\mathrm{vdW}}+\zeta-$ the local film thickness given by the global van der Waals equilibrium thickness $d_{\mathrm{vdW}}$ and the roughness-induced modulation $\zeta$.

When proceeding in the same way as for helium films on rough surfaces, ${ }^{8}$ 
we get for any undulation of the substrate

$$
\delta(x)=\sum_{j=-\infty}^{\infty} \delta_{j} \exp \left[\mathrm{i} k_{j} x\right] \quad \text { where } \quad \delta_{0}=0
$$

with $k_{j}=j 2 \pi / l, l$ being the lateral dimension of the substrate. The corresponding undulation of the film surface in first order is

$$
\zeta^{(1)}(x)=\sum_{j=-\infty}^{\infty} \zeta_{j}^{(1)} \exp \left[\mathrm{i} k_{j} x\right]
$$

with

$$
\zeta_{j}^{(1)}=\frac{\delta_{j}}{1+\frac{1}{3} k_{j}^{2} R d_{\mathrm{vdW}}}
$$

and, in particular, $\zeta_{0}^{(1)}=0$. In correspondence to the case of the helium films ${ }^{8}$ we get for the Laplace radius

$$
R=\frac{\gamma V_{\mathrm{a}}}{\Delta \mu}=\frac{\gamma V_{\mathrm{a}}}{-k_{\mathrm{B}} T \ln \left[p / p_{\mathrm{sat}}\right]} .
$$

Expanding the zeroth Fourier-coefficient up to the second order, one gets

$$
\zeta_{0}^{(2)}=\left\langle\frac{2}{9} R^{2} d_{\mathrm{vdW}}\left(\sum_{j=-\infty}^{\infty} k_{j}^{2} \zeta_{j}^{(1)} \exp \left[\mathrm{i} k_{j} x\right]\right)^{2}\right\rangle=\frac{1}{9} R^{2} d_{\mathrm{vdW}} \sum_{j=-\infty}^{\infty} k_{j}^{4} \zeta_{j}^{(1) 2}
$$

as the global thickening of the film caused by roughness. The temperature dependence herein is entirely hidden in $R$ and $d_{\mathrm{vdW}}$.

Putting everything together for a single Fourier-component $j$, the right side of Eq. 11 becomes

$$
\left[\frac{\frac{1}{3} k_{j}^{2} R \sqrt{d_{\mathrm{vdW}}} \delta_{j}}{1+\frac{1}{3} k_{j}^{2} R d_{\mathrm{vdW}}}\right]^{2}=\left[\delta_{j} \frac{\frac{1}{3} k_{j}^{2} \gamma V_{\mathrm{a}} \sqrt[5]{\Delta C_{3} k_{\mathrm{B}} / \Delta \mu^{7}}}{1+\frac{1}{3} k_{j}^{2} \gamma V_{\mathrm{a}} \sqrt[3]{\Delta C_{3} k_{\mathrm{B}} / \Delta \mu^{4}}}\right]^{2},
$$

which is a function of $\Delta \mu$ and the material constants only. Under the assumptions of the theory, there is no temperature dependence for $d$ at fixed chemical potential. For fixed $p / p_{\text {sat }}$ the film thickness decreases with temperature as it does for the FHH theory. Possible contributions due to changes in $\gamma$ and the molecular volume are, however, not yet taken into account.

Figure 3 shows the result of applying the roughness theory to one of our data-sets using three Fourier components. The roughness parameters have been adjusted to reproduce the shape of the measurement with good agreement. However, comparing the resulting surface profile to a typical measured $\mathrm{Au}$ substrate it appears unrealistic. In the case of a decrease in $\gamma$, the surface profile would have to be even steeper to achieve the same film thickness, so the general trend of decreasing surface tension towards the critical point leads to a decreasing film thickness with increasing temperature. 


\section{EXPERIMENTS}

\subsection{Experimental Setup and Procedure}

Our experiments have been carried out using a $\mathrm{Cu}$ sample cell cooled down in a helium gas-flow cryostat with optical access. The thickness of a hydrogen film adsorbed onto a gold film as substrate, which has been evaporated onto a glass prism, is measured by means of surface plasmon spectroscopy. ${ }^{10}$ The hereby measured effective thickness of the covering adsorbate has a resolution of $<1 \AA$.

Adsorption isotherms have been taken at different temperatures above and below the triple-point of hydrogen $T_{3}=13.9 \mathrm{~K}$ using the same substrate. A gas flow controller is used to achieve a very slow and steady increase of the pressure inside the cell, and no significant relaxation has been observed upon pausing throughout the adsorption. Also, at a single temperature the adsorption rate has been changed by a factor of four without significant influence. The total adsorption times were several hours.
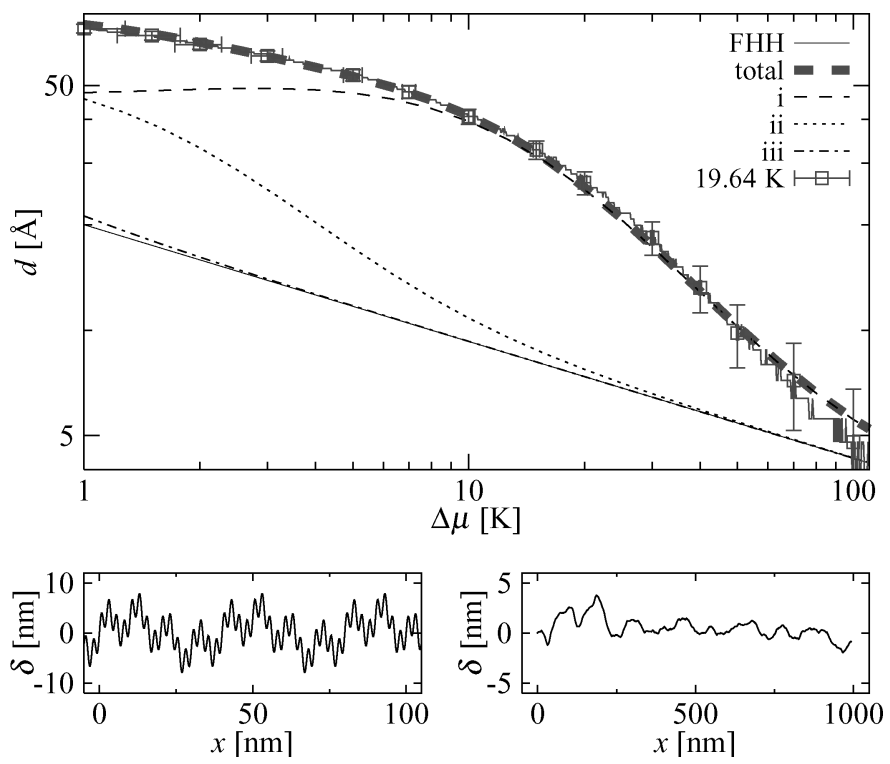

Fig. 3. Top: Application of the roughness model ${ }^{8}$ to the data measured at $19.64 \mathrm{~K}$ using 3 Fourier components: i) $\delta=2.4 \mathrm{~nm}, \lambda=2.5 \mathrm{~nm}$, ii) $\delta=3 \mathrm{~nm}, \lambda=10 \mathrm{~nm}$, iii) $\delta=3 \mathrm{~nm}, \lambda=40 \mathrm{~nm}$. Here $\gamma=2.17 \mathrm{~K}^{-2}$ (Ref. 6). The calculated curve (thick dashed line) reproduces the data very well. The overestimation at $\Delta \mu=100 \mathrm{~K}$ is due to the neglectance of the Brunauer-Emmett-Teller (BET) theory. ${ }^{9}$ Bottom: Comparison of the roughness profile as required for the fit (left) and a typical profile of a $50 \mathrm{~nm} \mathrm{Au} \mathrm{film} \mathrm{as} \mathrm{measured} \mathrm{by} \mathrm{AFM} \mathrm{(right).}$ 


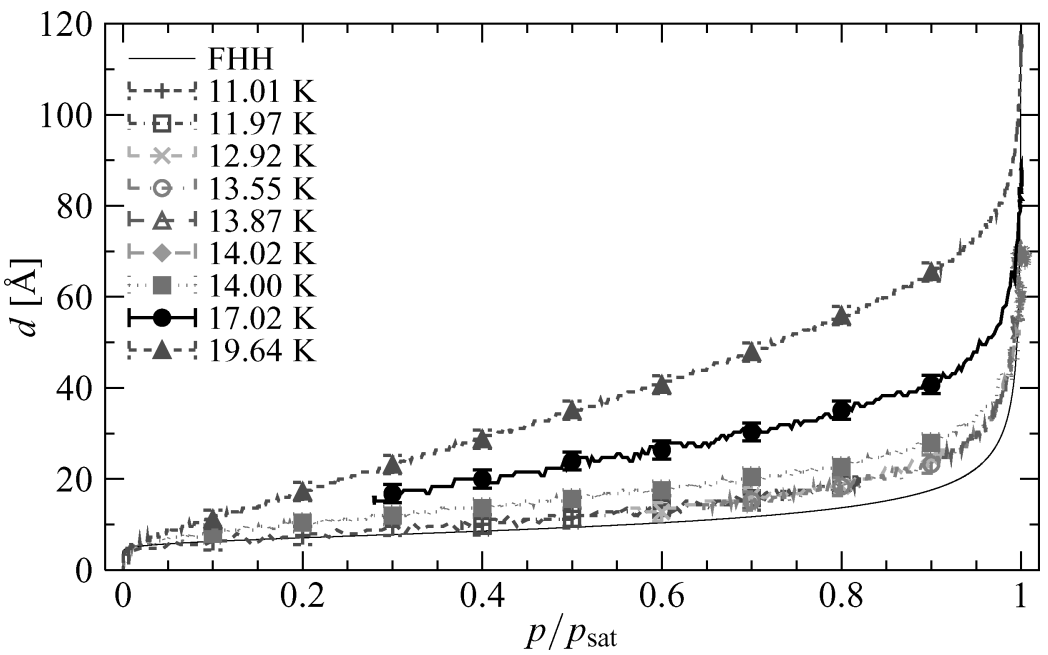

Fig. 4. Measured adsorption isotherms at several temperatures plotted as effective film thickness $d$ versus reduced pressure $p / p_{\text {sat }}$, where $p_{\text {sat }}$ is the saturated vapor pressure of the liquid phase at the corresponding temperature. The thin solid line shows the behavior as expected by the FHH theory at $14 \mathrm{~K}$, for a typical $\Delta C_{3}=$ $8000 \mathrm{~K} \AA^{3}$. The lowest measured curve consists of several isotherms below $T_{3}$ that, upon normalization, lie on each other. The measured film thicknesses are higher than predicted almost throughout the whole measurement range. This deviation strongly increases with temperature. For clarity, only few data-sets are shown for $T>T_{3}$.

\subsection{Experimental Results}

All our adsorption isotherms show deviations from the expectations according to FHH. These deviations grow with increasing temperature (see Figs. 4 and 5). Comparing these with the previously shown theoretical results (Figs. 1, 2, and 3) it is obvious that the measured film thicknesses cannot be explained by the above theories. Figure 5 clearly shows that the dependence is not linear as expected by FHH on this scale. The shape of excess thickness appears similar to the predictions by substrate roughness and thermal fluctuations. However, in the roughness case, the film thickness at given chemical potential is not a function of temperature, and thermal fluctuations only show strong thickness increase for very high temperatures, where the base $(\mathrm{FHH})$ thickness is very small.

In Figure 6 we present the temperature dependence of the effective film thickness while keeping a fixed $\Delta \mu$, as extracted from our measurements. For large $\Delta \mu$, a change in temperature has none or small influence only, while for small values of $\Delta \mu$ there is a significant increase (by a factor of 2 or more) for $T>T_{3}$. 


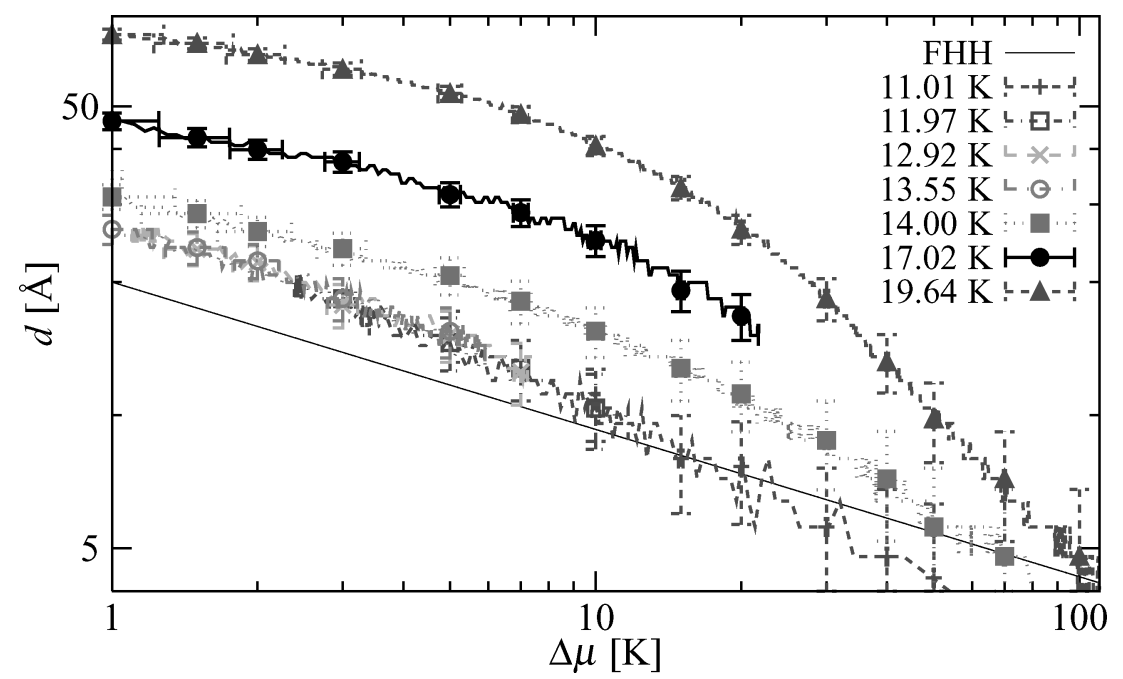

Fig. 5. Adsorption isotherms of Fig. 4 plotted as $\log d$ versus $\log \Delta \mu$. The straight solid line shows the behavior as expected by the FHH theory for $\Delta C_{3}=8000 \mathrm{~K} \AA^{3}$. For large values of $\Delta \mu$ the measured thickness is smaller than predicted due to crossover to the BET regime.

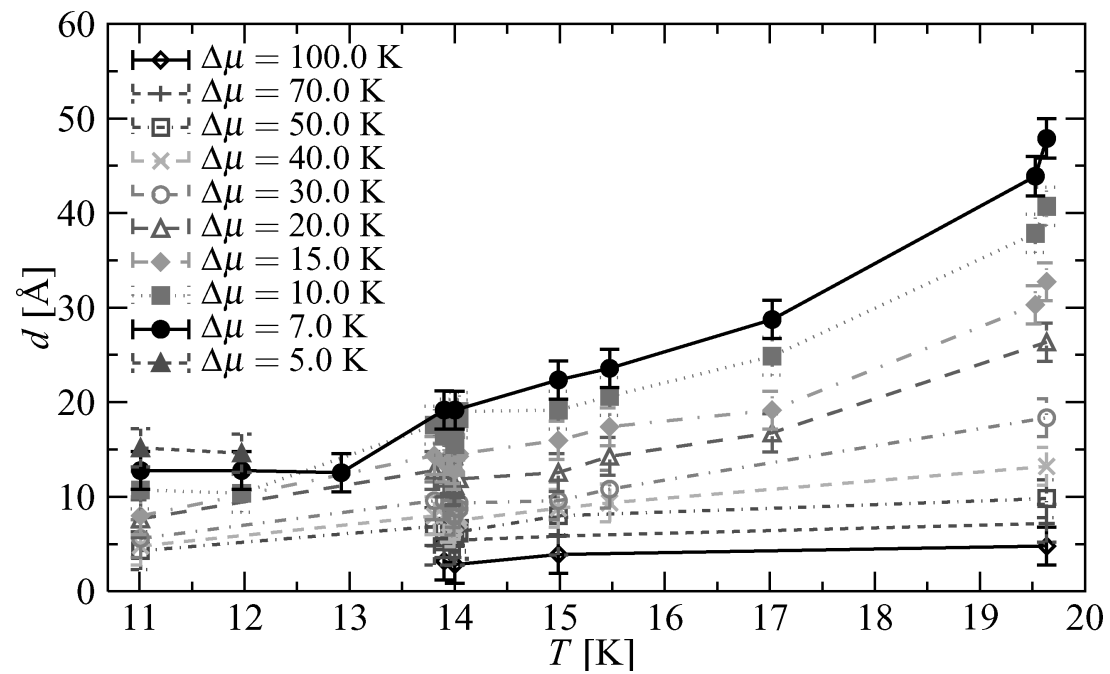

Fig. 6. Effective film thickness $d$ at given chemical potentials $\Delta \mu$ as function of temperature $T$. Only data with uncertainties up to $4 \%$ have been taken into account. For large $\Delta \mu(50-100 \mathrm{~K})$ there is none or only a small temperature dependence, while for $\Delta \mu$ is $7 \mathrm{~K}$ and $5 \mathrm{~K}$ the thickness increases by a factor of 2 or more when going from $T_{3}$ to $T=19.6 \mathrm{~K}$. Around $T_{3}$ the data points overlap within their error-bars. 


\section{CONCLUSIONS}

We have shortly introduced two theoretical approaches to extend the FHH theory in order to account for real adsorption substrates and compared them with a series of measurements of hydrogen on gold. It has become obvious that both cannot explain the experimental results by themselves, as they lack the experimentally found temperature dependence and/or the extent of thickness increase is (strongly) underestimated. It appears necessary to review and incorporate both aspects (substrate roughness and thermal fluctuations) into a single model and possibly even introduce additional mechanisms not taken into account so far in order to obtain quantitative agreement with our experimental data.

\section{ACKNOWLEDGMENTS}

We thank V. Shikin and K. Mecke for discussions. This work was supported by the DFG-Schwerpunkt "Wetting and Structure Formation at Interfaces" under grant Le 315/20.

\section{REFERENCES}

1. J. Frenkel, Kinetic Theory of Liquids, Clarendon, Oxford (1946).

2. G. Halsey, J. Chem. Phys. 16, 931 (1948).

3. T.L. Hill, J. Chem. Phys. 17, 520 (1949); ibid. 17, 590 (1949); ibid. 17, 668 (1949).

4. K.R. Mecke and J. Krim, Phys. Rev. B 53, 2073 (1995).

5. J. Vorberg, S. Herminghaus, and K. Mecke, Phys. Rev. Lett. 87, 196105 (2001).

6. J. Vorberg, Ph.D. Thesis, University of Potsdam (1997).

7. From Ref. 6: E.g., assuming the numerical values are for $32.26 \mathrm{~K}$ but scaling $\Delta \mu$ to $p / p_{\text {sat }}$ with $13.96 \mathrm{~K}$.

8. J. Klier, M. Zech, A. Fubel, P. Leiderer, and V. Shikin, J. Low Temp. Phys. 138, 355 (2005).

9. S. Brunauer, P.H. Emmett, and E. Teller, J. Am. Chem.Soc. 60, 309 (1938).

10. S. Tibus, M. Sohaili, J. Klier, and P. Leiderer, Low Temp. Phys. 29, 970 (2003). 